\title{
CL60 车轮材料表面激光离散熔凝的滚动磨损形貌
}

\author{
邢旭辉 ${ }^{1} \quad$ 李正阳 ${ }^{1} \quad$ 杨明江 ${ }^{1} \quad$ 占 剑 $^{1} \quad$ 林化强 \\ 中国科学院力学研究所, 北京 100190 \\ ( 2 青岛四方机车车辆股份有限公司高速列车系统集成国家工程实验室, 山东 青岛 266111)
}

\begin{abstract}
摘要 针对激光离散熔凝后的 CL60 材料磨损后的形貌问题, 使用 MM-P2 摩擦磨损试验机对 Nd… YAG 激光离散 熔凝后的圆环试样进行磨损试验, 使用 micro XAM 三维白光干涉表面形貌仪对磨损试验前后的试样表面进行测 量。使用金相显微镜和扫描电镜研究磨损试验后的试样金相。结果表明, 经过激光离散熔凝的点硬度高, 在磨损 过程中形成凸起; 熔凝点可以有效阻止试样表面塑性变形的积累, 提高材料整体的抗塑性变形能力, 减小试样表面 由于塑性变形而产生的层片状剥落, 表现出较高的耐磨性; 不同螺距点距的熔凝点分布可以形成不同的磨损后表 面形貌。
\end{abstract}

关键词 激光技术; CL60;激光离散熔凝; 磨损; 表面形貌

中图分类号 TN249;TG115. $5^{+} 8$ 文献标识码 A doi: 10.3788/CJL201340.0303006

\section{Rolling Wear Properties of CL60 Steel after Pulsed Nd: YAG Laser Surface Melting}

\author{
Xing Xuhui ${ }^{1}$ Li Zhengyang ${ }^{1}$ Yang Mingjiang ${ }^{1}$ Zhan Jian $^{1}$ Lin Huaqiang ${ }^{2}$ \\ ${ }^{1}$ Institute of Mechanics, Chinese Academy of Sciences, Beijing 100190, China \\ National Engineering Laboratory for High-Speed Train System Integration, CRS SiFang Co. , Ltd, \\ Qingdao, Shandong 266111, China
}

\begin{abstract}
Laser surface melting is carried out using a pulsed Nd $\cdots$ YAG laser on specimens made of CL60 steel, after that the rolling wear properties of specimens are studied using a MM-P2 rolling friction and wear test machine. The surface topography of the specimens before and after the rolling wear test is measured by a micro XAM 3D surface topography instrument. Metallographic examinations of the specimens are taken out after the rolling wear test using metallographic microscope and scanning electron microscope. It turns out that the laser melted spots stand out during the experiments due to its higher hardness. The laser melted spots are not only reducing the plastic deformation themselves, but also hindering the plastic deformation of vicinity base material on the same circle. The laser melted spots can reduce the scraps peeling off from specimens and the rolling wear resistance is improved. Different distributions of laser melted spots can form different surface topographies after wear.
\end{abstract}

Key words laser technique; CL60; pulsed laser surface melting; wear; surface topography

OCIS codes $140.3390 ; 350.3390 ; 350.3850$

\section{1 引言}

激光离散熔凝在工业上得到越来越多的应用。 激光离散熔凝的方法可以大大加快加工速度且耐磨 性和连续激光处理相差不大 ${ }^{[1]}$ 。激光离散熔凝在工 件上形成一种性能不均匀的表面, 在磨损过程中会
形成一定分布的表面形貌; 由于这些熔凝点具有一 定深度,表面的形貌可以维持较长时间。这些熔凝 点可以改善材料的抗磨损性能,形成的稳定表面形 貌又可以改变材料的摩擦。国内外已经有用脉冲激 光对耐热合金 ${ }^{[2,3]}$ 、可锻铸铁 ${ }^{[4]}$ 、硬质合金 ${ }^{[5]}$ 、高速

收稿日期: 2012-09-14; 收到修改稿日期: 2012-11-27

基金项目：国家科技支撑计划(2009BAG12A01-B10-2)资助课题。

作者简介: 邢旭辉(1983-), 男, 博士研究生, 主要从事激光表面处理方面的研究。E-mail: xingxuhui@gmail. com

导师简介: 杨明江 (1944一), 男, 研究员, 博士生导师, 主要从事激光表面处理方面的研究。E-mail: ymj1@imech. ac. cn 
光

钢 ${ }^{[6]}$ 、不锈钢 ${ }^{[7]}$ 、模具钢 ${ }^{[8]}$ 等材料进行表面局部熔凝 处理的实验报道。但以往的激光离散熔凝的研究都 主要集中在熔凝层的组织变化、性能变化方面, 未见 有将离散熔凝后的材料作为整体对其磨损后的表面 形貌变化进行研究的报道。要研究激光离散熔凝对 材料摩擦的影响需要对离散熔凝后的材料在磨损后 的表面形貌进行分析。

本文针对 CL60 车轮材料, 使用 YAG 激光器在 试样表面进行离散熔凝, 在 MM-P2 滚动摩擦磨损
试验机上对试样进行了试验, 并使用 micro XAM 三 维白光干涉表面形貌仪对试验前后的试样表面形貌 进行检测, 研究其磨损行为; 对试验后的试样剖面在 金相显微镜和扫描电镜下进行观察, 分析形成磨损 后表面形貌的原因。

\section{2 试验材料与方法}

CL60 车轮材料和作为对磨试样的 U71Mn 钢 轨材料,其化学成分如表 1 所示。

表 1 材料成分 (质量分数, $\%$ )

Table 1 Material composition (mass fraction, \%)

\begin{tabular}{cccccc}
\hline Material & $\mathrm{C}$ & $\mathrm{Si}$ & $\mathrm{Mn}$ & $\mathrm{S}$ & $\mathrm{P}$ \\
\hline $\mathrm{CL} 60$ & $0.65 \sim 0.76$ & $0.15 \sim 0.35$ & $1.10 \sim 1.40$ & $\leqslant 0.03$ & $\leqslant 0.03$ \\
$\mathrm{U} 71 \mathrm{Mn}$ & $0.55 \sim 0.65$ & $0.17 \sim 0.37$ & $0.5 \sim 0.8$ & $\leqslant 0.03$ & $\leqslant 0.03$ \\
\hline
\end{tabular}

如图 1 所示, CL60 和 U71Mn 试样尺寸一致, 直径为 $45.4 \mathrm{~mm}$, 接触部分宽度为 $5 \mathrm{~mm}$ 。采用 YAG 激光器, 对 CL60 试样进行激光离散熔凝。通 过反复试验优化的工艺参数如表 2 所示, 采用了如 图 2 所示激光波形,高功率密度的激光波形前沿能 迅速提高钢铁表面的温度, 提高钢铁表面对激光的 吸收率, 又不至于太高而使试样表面大量汽化或产 生等离子体而屏蔽激光能量; 后续激光功率降低, 可

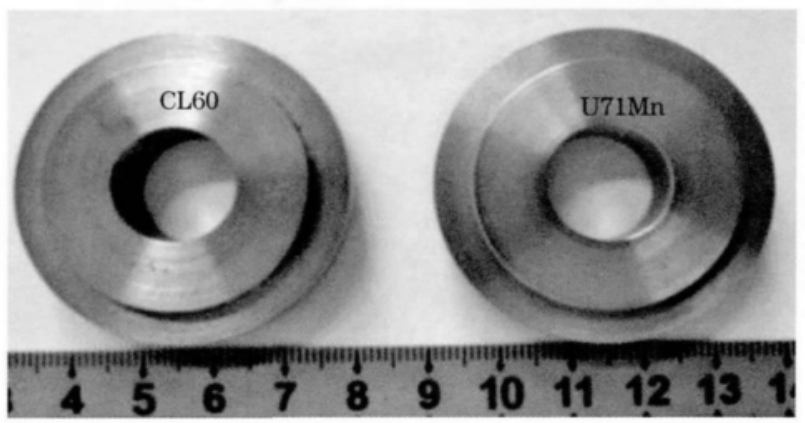

图 1 CL60 和 U71Mn 试样

Fig. 1 Specimens of CL60 and U71Mn

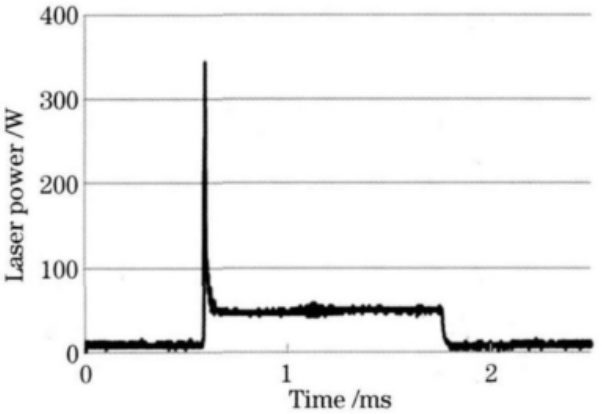

图 2 激光波形

Fig. 2 Waveform of laser
在改造后的 MM-P2 试验机上对处理后 CL60

以稳定地给熔池提供能量, 保持熔池温度, 促进熔池 的生长, 又不至于使熔池能量积累过多而发生汽化, 使熔池从激光获得的能量与通过热传导损失的能量 达到平衡 ${ }^{[9]}$; 脉冲宽度较宽, 可以得到足够的熔凝深 度。试样表面激光分三种分布处理,矩形分布 $a 、 b$ 和菱形交叉分布 $\mathrm{c}$, 其分布参数如表 3 所示。图 3 中,三种分布的单位面积点数量相同。
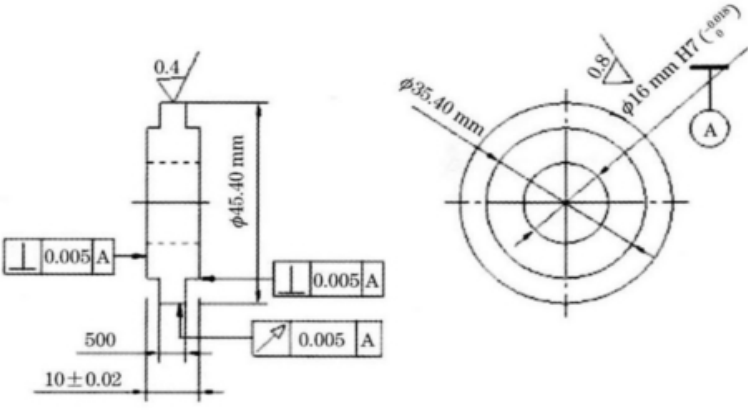
试样进行磨损试验, U71Mn 钢轨试样作为对磨材 料,载荷为 $800 \mathrm{~N}$, 摩擦系数为 0.2 , 磨损时间为 $2 \mathrm{~h}$, 转速为 $400 \mathrm{r} / \mathrm{min}$ 。使用 micro XAM 三维白光干涉 表面形貌仪对试验前后的试样表面形貌进行检测, 研究其磨损行为; 对试验后的试样在金相显微镜和 扫描电镜下进行金相分析。

利用数值计算的方法对假想试样上存在凸起的 情况分析加载后的接触应力分布。由于试样之间是 线接触, 认为在接触斑的非边缘区域具有对称性, 只 取试样上的一个表面形貌重复单元进行计算。接触 
斑尺寸相对于试样的曲率半径很小, 计算接触应力时可以等效成一个椭球面和一个平面接触。

\section{表 2 激光参数}

Table 2 Parameters of laser

\begin{tabular}{ccccccc}
\hline $\begin{array}{c}\text { Pulse } \\
\text { width } / \mathrm{ms}\end{array}$ & $\begin{array}{c}\text { Pulse } \\
\text { energy } / \mathrm{J}\end{array}$ & $\begin{array}{c}\text { Peak } \\
\text { power } / \mathrm{W}\end{array}$ & $\begin{array}{c}\text { Average } \\
\text { power } / \mathrm{W}\end{array}$ & $\begin{array}{c}\text { Spot } \\
\text { diameter } / \mu \mathrm{m}\end{array}$ & $\begin{array}{c}\text { Peak power } \\
\text { density } /\left(\mathrm{W} / \mathrm{cm}^{2}\right)\end{array}$ & $\begin{array}{c}\text { Average power } \\
\mathrm{density} /\left(\mathrm{W} / \mathrm{cm}^{2}\right)\end{array}$ \\
\hline 1.2 & 0.072 & 348.2 & 48 & 120 & $3.14 \times 10^{6}$ & $4.34 \times 10^{5}$ \\
\hline
\end{tabular}

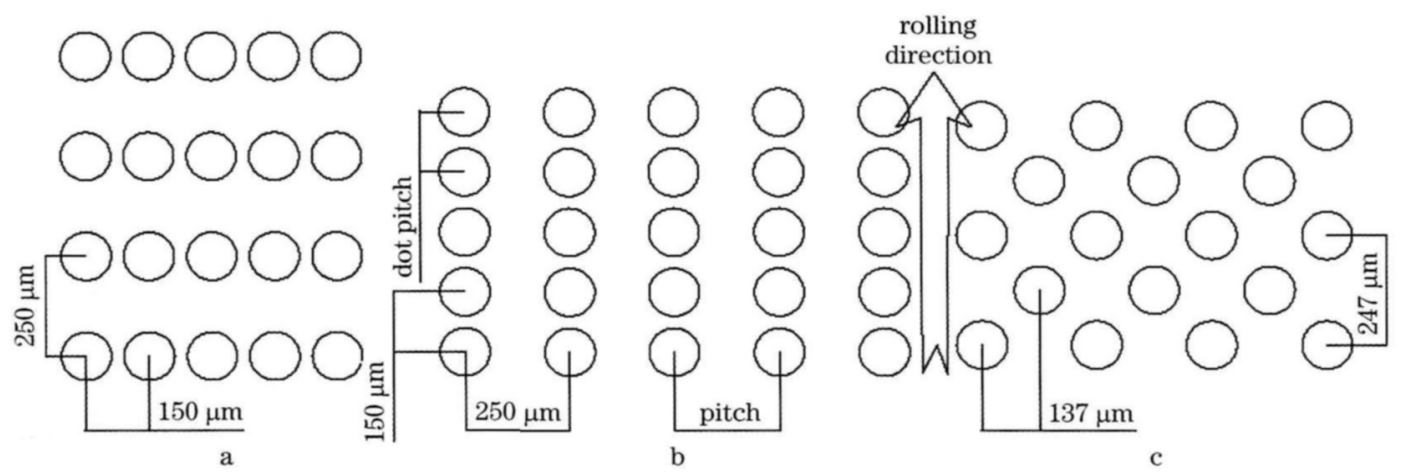

图 $3 a$ a b 、 三种试样分布的示意图

Fig. 3 Schematics of $a, b$ and $c$ specimens distributions

表 3 试样表面激光熔凝点的分布

Table 3 Distributions of laser melted spots on surface of specimens

\begin{tabular}{cccc}
\hline Distribution & a & b & c \\
\hline Pitch $/ \mu \mathrm{m}$ & 150 & 250 & 137 \\
Dot pitch $/ \mu \mathrm{m}$ & 250 & 150 & 274 \\
\hline
\end{tabular}

\section{3 实验结果与分析}

使用 $\mathrm{MH}-6$ 显微硬度计测量基体材料硬度为 $331 \mathrm{HV}$, 熔凝点硬度为 $757 \mathrm{HV}$ 。使用 micro XAM 三维白光干涉表面形貌仪对磨损试验前后的试样表 面进行测量。

\section{1 试样表面的磨损特征}

激光离散熔凝处理会在试样表面形成经过强化 的圆周环带和未经强化的圆周环带交替分布 (如 图 4 所示)。滚动磨损中,犁沟效应、表面塑性变形 都是沿着圆周带方向的, 经过强化的圆周带上分布 着高硬度的强化点, 能更好地抵抗抗磨粒磨损和表 面塑性变形,使得经过强化的圆周带磨损小而相对 凸起, 试样表面出现沿圆周方向的条纹。图 5 是三 种分布的激光离散熔凝工艺的试样在磨损试验前后 三维照片的对比, 试样的滚动方向如图所示。可以 看出, 当激光离散熔凝点的点距分布较大时 [图 5(a)], 经过激光离散熔凝处理的点在磨损后形 成凸起点, 点的一侧有大量的片状堆积物 [图 6(a) 中的 I区]。当点距较小时 [图 5(b)], 经过激光离
散熔凝处理的点在滚动方向上互相连接成条纹并明 显凸起, 条纹间距与螺距相等。激光离散熔凝处理 后的表面在磨损中并不仅仅是经过熔凝后的高硬度 组织由于耐磨损而凸起, 而且凸点会承担主要的载 荷以及抗磨损的任务 ${ }^{[10]}$, 使整个经过强化的圆周环 带上的磨损减小。

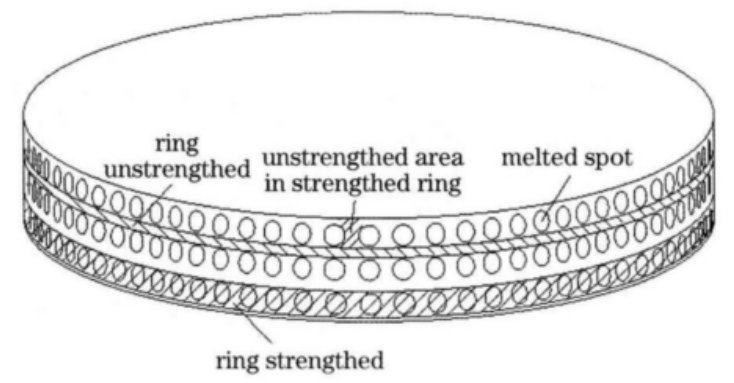

图 4 激光离散熔凝处理后试样的强化和未经 强化的圆周环带

Fig. 4 Strengthed and unstrengthed rings on the surface of laser melted specimens

图 5 中试样 $\mathrm{a} 、 \mathrm{~b} 、 \mathrm{c}$ 的表面形貌参数如表 4 所 示。最高凸起与最低的凹陷(忽略毛刺)高度差分别 为 $7 、 15.4 、 3 \mu \mathrm{m}$ 。说明在相同的熔凝点分布密度 下, 减小螺距可以降低末经强化的圆周环带的磨损。 当激光离散熔凝点的分布螺距较大时, 条纹之间形 成很深的凹谷, 螺距越大, 条纹之间的凹陷越深, 凹 陷处的片状剥落物越明显 [图 6(a)、(b)中的 II 区、 III 区]; 当螺距较小时, 条纹之间的凹陷深度较小,凹 陷处无明显的剥落痕迹。 
表 4 试样 $\mathrm{a} 、 \mathrm{~b} 、 \mathrm{c}$ 的表面形貌参数

Table 4 Surface topography of the specimens a, b and c

\begin{tabular}{cccc}
\hline Distribution & $\mathrm{a}$ & $\mathrm{b}$ & $\mathrm{c}$ \\
\hline$R_{\mathrm{a}}$ before wear $/ \mu \mathrm{m}$ & 1.41 & 2.98 & 1.58 \\
$R_{\mathrm{a}}$ after wear $/ \mu \mathrm{m}$ & 1.79 & 3.74 & 0.59 \\
Pitch $/ \mu \mathrm{m}$ & 150 & 250 & 137 \\
Width of stripe $/ \mu \mathrm{m}$ & 150 & 250 & 137 \\
Dot pitch $/ \mu \mathrm{m}$ & 250 & 150 & 274 \\
Height of stripe $/ \mu \mathrm{m}$ & 7 & 15.4 & 3 \\
\hline
\end{tabular}

熔凝点的交错分布可以有效地避免试样上出现
较宽的未经强化的圆周环带, 这些环带在摩擦中受 到严重的磨损，所以交错分布可以明显地减小磨损。 图 5(c) 和图 6(c) 中的菱形分布处理后的试样在磨 损试验后没有观察到表面的片状剥落。图 6(a)、 （b）中表面的片状剥落物属于严重磨损过程, 塑性 变形 裂纹萌生一裂纹扩展一片状剥落 ${ }^{[11 \sim 13]}$ 中的最后 一步,这些表面剥落的现象在很多磨损行为中被观 察到 ${ }^{[14 \sim 16]}$, 阻止或减轻表面剥落过程可以有效地降 低磨损。

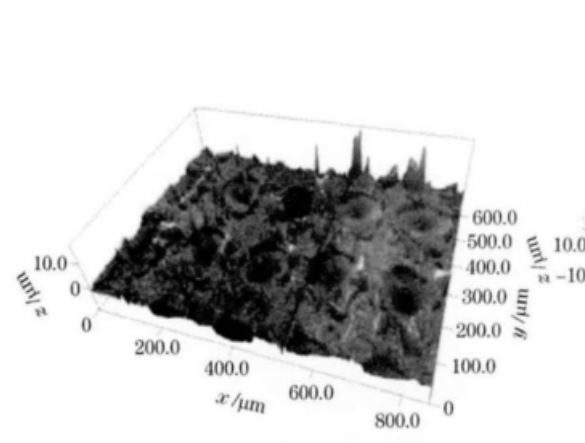

(a1)

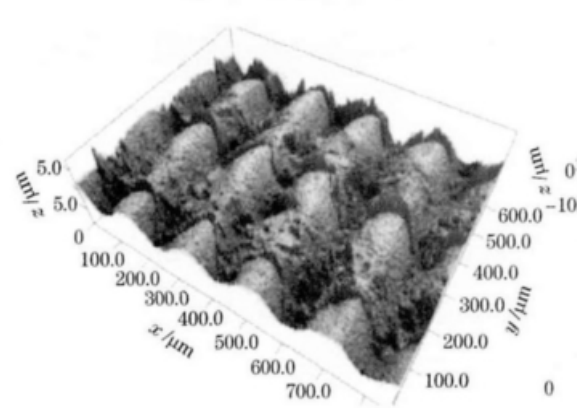

(a2)

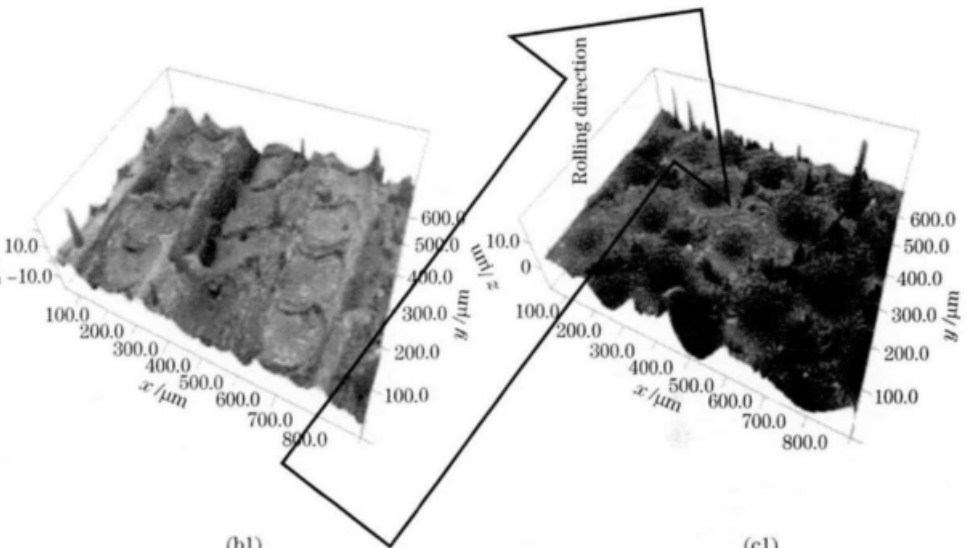

(b1)

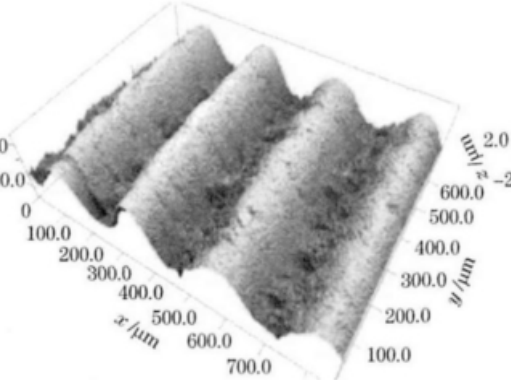

(b2)

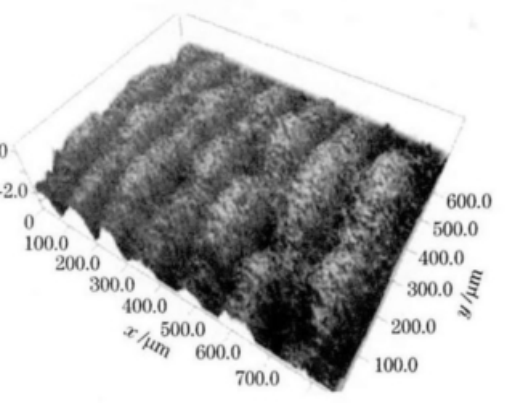

(c2)

图 5 试样 $\mathrm{a} 、 \mathrm{~b} 、 \mathrm{c}$ 的磨损试验前 $[(\mathrm{a} 1),(\mathrm{b} 1),(\mathrm{c} 1)]$ 和后 $[(\mathrm{a} 2),(\mathrm{b} 2),(\mathrm{c} 2)]$ 三维照片

Fig. 5 3D photos of specimens a,b and c before $[(a 1),(b 1),(c 1)]$ and after $[(a 2),(b 2),(c 2)]$ wear experiments
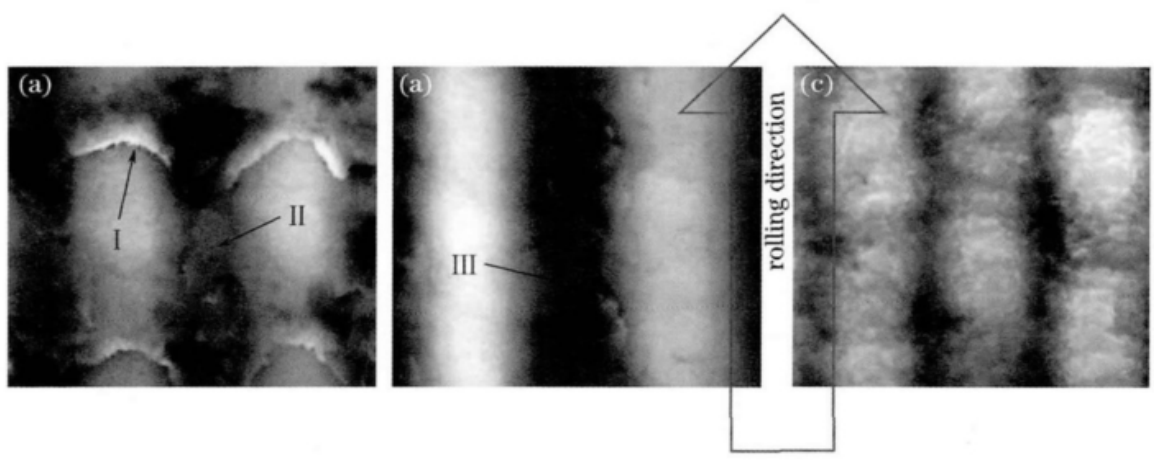

图 6 试样 a (a)、b (b)、c (c) 的磨损实验后二维照片

Fig. 6 2D photos of specimens (a) a、(b) b and (c) c after wear experiments 
对试样表面激光离散熔凝点的分布和磨合后产 生的条纹高度与宽度之间的关系进行进一步研究。 可以明显看出条纹宽度总是和激光离散熔凝分布的 螺距相等 (如表 4 所示)。使用激光离散熔凝处理一
批试样, 其表面熔凝点为矩形分布, 分成两组, 第一 组固定螺距改变点距, 第二组固定点距改变螺距。 对这些试样磨合后测量其条纹高度,结果如表 5 所示。

表 5 螺距、点距与条纹高度的关系

Table 5 Relationship among pitch, dot pitch and height of stripe

\begin{tabular}{cccccccc}
\hline Pitch $/ \mu \mathrm{m}$ & 200 & 200 & 200 & 200 & 200 & 200 & 200 \\
Dot pitch $/ \mu \mathrm{m}$ & 125 & 150 & 175 & 200 & 225 & 250 & 275 \\
Height of stripe $/ \mu \mathrm{m}$ & 13.1 & 13 & 12.2 & 12.1 & 10.3 & 9.2 & 6.7 \\
\hline Pitch $/ \mu \mathrm{m}$ & 125 & 150 & 175 & 200 & 225 & 250 & 275 \\
Dot pitch $/ \mu \mathrm{m}$ & 200 & 200 & 200 & 200 & 200 & 200 & 200 \\
Height of stripe $/ \mu \mathrm{m}$ & 7.2 & 9 & 11.5 & 12.1 & 12.8 & 13.2 & 13.3 \\
\hline
\end{tabular}

图 7 是根据表 5 中的数据得到的曲线,曲线分 别表示固定螺距时条纹高度随着点距的变化, 以及 固定点距时条纹高度随着螺距的变化。

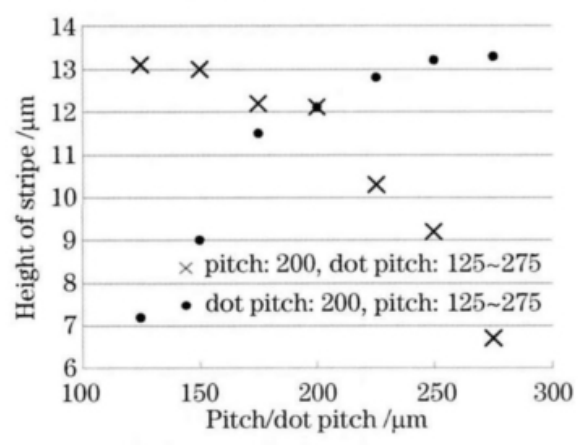

图 7 螺距、点距与条纹高度的关系

Fig. 7 Relationship among pitch, dot pitch and stripe height

从图 7 可以看出, 当螺距固定时条纹高度随着 点距的减小而增大, 当点距减小到 $200 \mu \mathrm{m}$ 以下时, 条纹高度随着点距的变化减慢。分析其原因是由于 随着点距的减小, 试样上被强化的圆周上熔凝点所 占的比例越来越大, 具有熔凝点的圆周抗塑性变形 耐磨损能力越来越强; 当点距小于 $200 \mu \mathrm{m}$ 时, 熔凝 点之间距离很近 (熔凝点的直径在 $150 \mu \mathrm{m}$ 左右), 此时试样上被强化的圆周抗塑性变形耐磨损能力已 经足够强, 继续减小直至熔凝点发生搭接, 抗塑性变 形耐磨损能力没有显著增强, 所以当点距减小到 $200 \mu \mathrm{m}$ 以下时,条纹高度随着点距的变化减慢。

当点距固定时条纹高度随着螺距的增大而增 大, 当螺距增大到 $225 \mu \mathrm{m}$ 以上时, 条纹高度随着螺 距的变化减慢。分析其原因是当强化后的环带在磨 损中凸起之后承担了更多的应力, 对相邻未强化环 带起到了保护作用; 随着螺距的增大试样上未经强 化的圆周环带变宽, 环带中远离两侧强化环带的地 方受到的保护较小, 所以随着螺距的增大未强化环 带的磨损深度增加, 等效于条纹高度增加; 当螺距增
大到 $225 \mu \mathrm{m}$ 以上时,未经强化的圆周环带已足够 宽, 未强化环带中心附近受到两侧强化环带的保护 已经较微弱, 继续增加未强化环带的宽度影响不再 显著, 所以当螺距增大到 $225 \mu \mathrm{m}$ 以上时, 条纹高度 随着螺距的变化减慢。

\section{2 试样表面的接触应力分布}

从试样表面的接触应力分布来分析观察到的现 象,对如图 8(a) 所示的表面形貌的试样加载后的接 触应力进行数值计算。分别计算了矩形分布螺距 $150 、 250 \mu \mathrm{m}$ 、点距 $250 、 150 \mu \mathrm{m}$, 菱形分布螺距 $137 \mu \mathrm{m}$ 、点距 $274 \mu \mathrm{m}$, 凸点直径 $150 \mu \mathrm{m}$, 凸点高度 $1.2 \mu \mathrm{m}$ 的几种表面的试样在加载 $800 \mathrm{~N}$ 的情况下 表面接触应力的分布, 得到的应力分布如图 8(b) 所 示。这些凸点在试样表面的分布密度相同, 面积占 有率都是 $47 \%$ 。数值计算取试样表面的一个重复 单元划分网格, 根据接触区域的变形协调, 采用牛顿 法进行收玫,试样表面形变根据弹性半空间物体在 法向集中力作用下的表面位移计算方法 ${ }^{[17]}$ 将面力 离散化, 每个网格受力后的位移可表示为

$$
d(x, y)=\frac{2 s}{\pi E^{\prime}} \sum_{x^{\prime}=1}^{x_{0}} \sum_{y^{\prime}=1}^{y_{0}} \frac{P\left(x^{\prime}, y^{\prime}\right)}{\sqrt{\left(x-x^{\prime}\right)^{2}+\left(y-y^{\prime}\right)^{2}}},
$$

式中 $P$ 为压强, $E^{\prime}$ 为试样的等效弹性模量, 表示为 $E^{\prime}=\frac{0.5 E}{1+v}, E$ 为试样的弹性模量, $u$ 为泊松比, $s$ 为单 位网格面积, $x_{0} 、 y_{0}$ 为 $x 、 y$ 方向网格数。

虽然具有凸点的地方应力要高于基体, 但是由 于熔凝点的高硬度, 并没有被磨平, 这一点从前文的 实验中得到了证实(图 5、图 6)。

从计算结果可以看到, 当螺距较小而点距较大 时, 沿试样滚动方向相邻凸点的凹陷处 [图 8(a1) 中 $\mathrm{A}$ 点]应力较大; 当螺距较大而点距较小时, 沿试样 

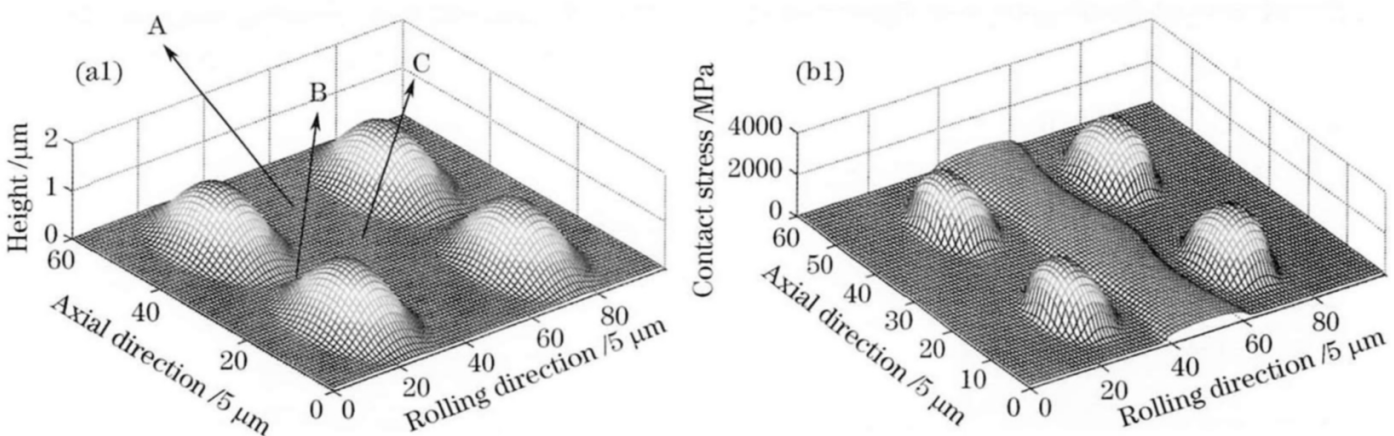

Pitch of $150 \mu \mathrm{m}$, dot pitch of $250 \mu \mathrm{m}$, the highest stress is $458.5 \mathrm{MPa}$ outside preiections
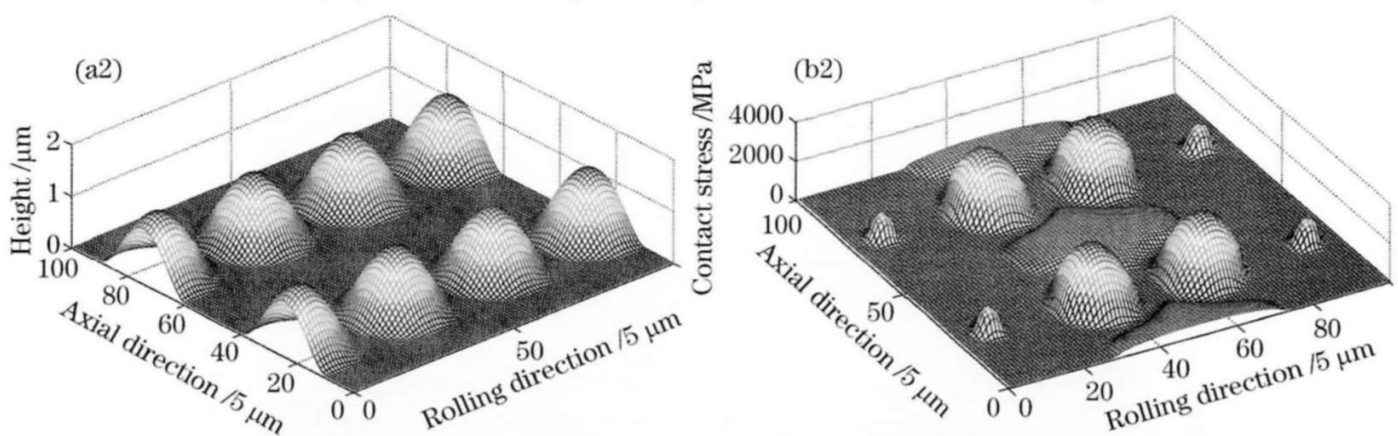

Pitch of $250 \mu \mathrm{m}$, dot pitch of $150 \mu \mathrm{m}$, the highest stress is $522.2 \mathrm{MPa}$ outside preiections
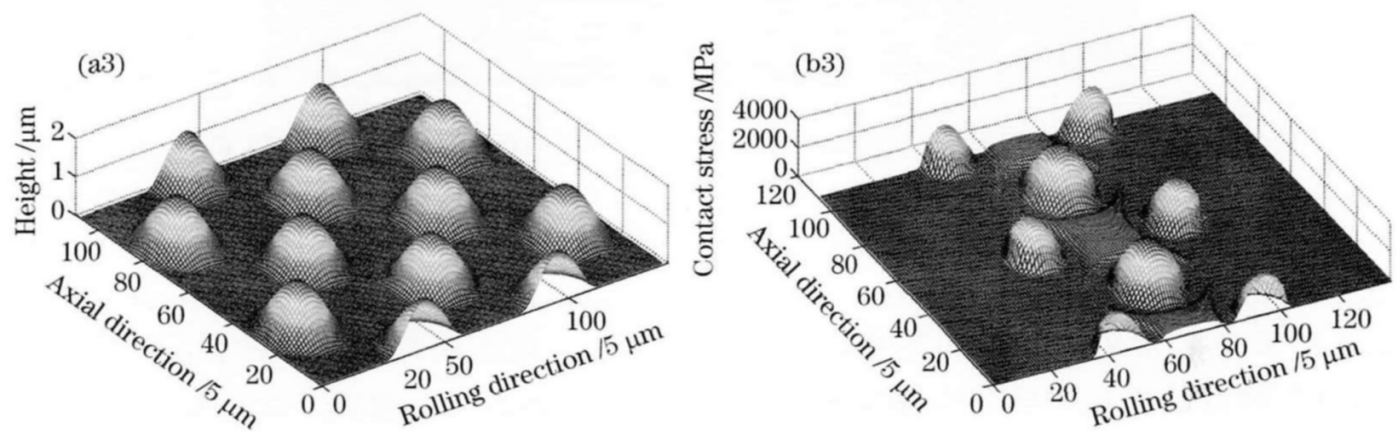

Pitch of $137 \mu \mathrm{m}$, dot pitch of $274 \mu \mathrm{m}$, the highest stress is $478.6 \mathrm{MPa}$ outside preiections

图 8 表面凸点形貌 $(\mathrm{a} 1) \sim(\mathrm{a} 3)$ 与表面应力分布 $(\mathrm{b} 1) \sim(\mathrm{b} 3)$

Fig. 8 Surface topography (a1) (a3) and contact stress distribution (b1) (b3)

轴线方向相邻凸点的凹陷处 [图 8(a1) 中 B 点]应力 较大; 而凸点外最大应力都出现在相邻四点的中间 [图 8(a1)中 C 点]。相同条件的材料, 接触应力较 大的地方磨损也较严重。计算得到的结果解释了 图 5 中试样 $\mathrm{a}$ 和 $\mathrm{b}$ 磨损后形貌的差别, 螺距大点距 小则 A 处磨损较轻微,试样表面容易形成条纹;螺 距小点距大则 B 处磨损较轻微, 试样表面容易形成 独立的凸点。

上述计算结果还未能完全解释图 6 中的现象, 图 6 中可以观察到无论点距螺距怎么变化, 试样表 面都有条纹产生, 在没有熔凝点分布的试样表面圆 周总是磨损严重形成凹槽。为了进一步分析其原 因, 分别观察无熔凝点和有熔凝点试样在磨损后的 断口金相。

\section{3 试样磨损后的表面塑性变形特点}

图 9(a) 是无激光作用车轮试样沿滚动方向的 断面, 表面形成的剪切塑性变形区是连续的。 图 9(b) 是激光离散熔凝区未磨完时车轮试样的沿 滚动方向断面, 从图中可见,塑性变形区被激光熔凝 区分隔开,激光熔凝区无明显塑性变形,在靠近左侧 激光熔凝区处,无明显塑性变形, 说明剪切力在遇到 高硬度的激光熔凝区时, 激光熔凝区不易发生塑性 变形 ${ }^{[17]}$, 而且可以阻碍附近基体组织塑性变形的累 积 ${ }^{[14]}$ 。这就解释了图 6(c)中没有观察到片状物的 原因,由于试样表面被熔凝点分割, 不存在大片的连 续基体组织,试样表面的塑形变形都在小范围内被 熔凝点阻止,塑性变形的积累被打断,没有发展到片 状录落的程度。 

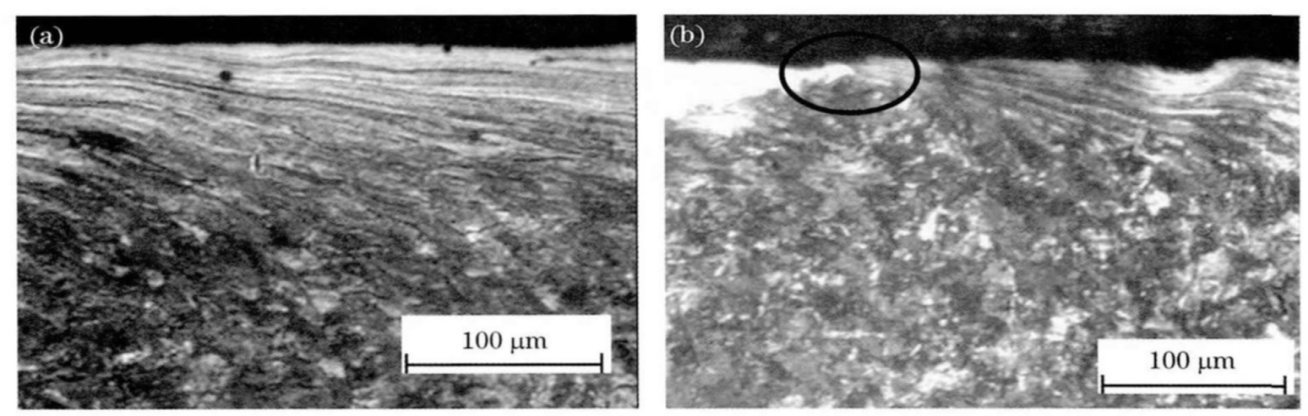

图 9 (a) 无激光作用及 (b) 激光离散熔凝 CL60 试样磨损试验后沿滚动方向断面

Fig. 9 Rolling direction section photomicrograph of CL60 specimens (a) without and (b) with laser melting after wear

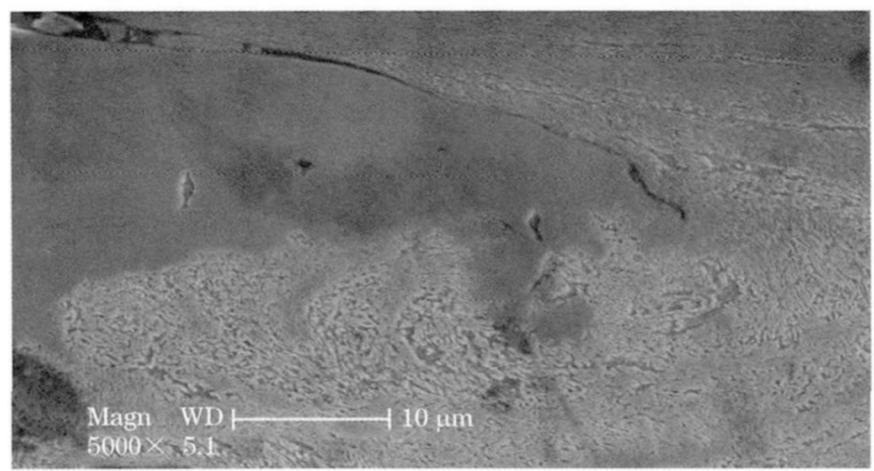

图 10 激光离散熔凝 CL60 试样表面磨损试验后沿滚动方向流线型塑性变形

Fig. 10 Plastic flow along the rolling direction after wear experiments in laser melted CL60 specimens

图 10 是扫描电镜下的图 9(b) 中左侧椭圆区域 中心不同组织交界处的图像, 可以观察到基体表面 的塑性变形在熔凝点处被阻挡而堆积到熔凝点表 面, 这些片状堆积物就是图 6(a) I 区中观察到的熔 凝点一侧的片状堆积物, 这些堆积物是由于基体的 塑性变形被熔凝点阻碍而堆积到熔凝点之上形成 的, 比片状剥落物更大更厚; 在熔凝点边缘附近形成 漩涡状的流线, 而熔凝点内部没有这种流线, 说明熔 凝点本身抗塑性变形的能力高于基体。

\section{4 结 论}

1）激光离散熔凝的熔凝点本身具有比基体高 很多的硬度, 基体硬度 $331 \mathrm{HV}$, 熔凝点硬度 $757 \mathrm{HV}$ 。高硬度的熔凝组织具有很高的抗塑性变 形能力, 在磨损过程中表现出极高的耐磨性, 会渐渐 凸起。

2) 由于激光离散熔凝点的支撑作用, 熔凝点周 边的基体受到的接触应力小于其他区域, 磨损也随 之减慢。

3）激光离散熔凝的熔凝点将材料表面的基体 组织分割成不连续的小区域时, 可以有效阻止试样 表面塑性变形的积累, 提高材料整体的抗塑性变形
能力,大大减小试样表面由于塑性变形而产生的层 片状剥落, 从而表现出较高的耐磨性。

4）在相同的熔凝点密度情况下, 熔凝点分布状 况对磨损后的形貌有重要影响。当熔凝点菱形排列 时,比矩形排列磨损后表面的起伏小, 表面形貌更为 均匀。

5）激光离散熔凝后的试样在磨合后会在表面 形成条纹, 条纹的宽度和熔凝点分布的螺距相等, 高 度随着螺距的增加和点距的减小而增大。

\section{参 考 文 献}

1 L. Xue, M. U. Islam, G. McGregor. Dot matrix hardening of steels using a fiber optic coupled pulsed Nd $\cdots$ YAG laser $[\mathrm{J}]$. Materials and Manufacturing Processes, 1999, 14(1): 53 65

2 B. S. Yilbas, M. Khaled, M. A. Gondal et al. . Nano-second pulse laser treatment of Incoloy $800 \mathrm{HT}$ alloy-corrosion properties [J]. Optics and Lasers in Engineering, 1999, 32(2): 157 172

3 Zhong Rutao, Xiong Zheng. Research on continuous/pulse laser cladding superalloy K403[J]. Chinese J. Lasers, 2010, 37 (3): $852 \sim 857$

钟如涛, 熊 征. 连续/脉冲 $\mathrm{CO}_{2}$ 激光熔覆高温合金 $\mathrm{K} 403[\mathrm{~J}]$. 中国激光, 2010, 37(3): 852 857

4 S. P. Gadag, M. N. Srinivasan. Dry sliding wear and friction: laser-treated ductile iron[J]. Wear, 1994, 173(1-2) : 21 29

5 S. I. Yares'ko. The influence of the composition of cobalt phase of hard alloys on tool wear upon laser hardening $[\mathrm{J}]$. Russian Journal of Non-Ferrous Metals, 2009, 50(5): 556 562 


中 国

$6 \mathrm{~J}$. KUSIŃSKI. Microstructure, chemical composition and properties of the surface layer of M2 steel after laser melting under different conditions $[J]$. Applied Surface Science, 1995, 86(1-4): $317 \sim 322$

7 U. Kamachi Mudali, M. G. Pujar, R. K. Dayal. Effects of laser surface melting on the pitting resistance of sensitized nitrogen-bearing type $316 \mathrm{~L}$ stainless steel $[\mathrm{J}]$. Materials Engineering and Performance, 1996, 5(1): 124 128

8 Huang Kaijin, Lin Xin, Xie Changsheng. Study of thin plate $012 \mathrm{Al}$ die steel during pulsed $\mathrm{Nd} \cdots \mathrm{YAG}$ laser melting $[\mathrm{J}]$. China Mechanical Engineering, 2006, 17(S1) : 327 330

黄开金, 林 金金, 谢长生. 薄板 $012 \mathrm{Al}$ 模具钢脉冲 $\mathrm{Nd} \cdots \mathrm{YAG}$ 激 光熔凝的研究 $[\mathrm{J}]$. 中国机械工程, 2006, 17(S1)：327 330

9 Zhan Jian, Yang Mingiiang, Wang Hongcai. Effect of YAG laser pulse waveform to laser etching crater morphology on cylinder wall[J]. Chinese J. Lasers, 2011, 38(6): 0603032

占 剑, 杨明江, 王红才. 脉冲 YAG 激光波形对缸套内壁激光 刻蚀微坑形貌的影响 $[\mathrm{J}]$. 中国激光, 2011, 38(6): 0603032

10 Li Meiyan, Wang Yong, Han Bin et al. . High-temperature wear property of high chrome steel by laser surface melting $[\mathrm{J}]$. Chinese J. Lasers, 2010, 37(1): 302 306

李美艳, 王 勇, 韩 涁等. 激光熔凝处理高铬钢的高温磨损性 能 $[\mathrm{J}]$. 中国激光, 2010, 37(1): 302 306

11 Shen Lian, Li Chenglao. Effect of laser melting processing on the microstructure and wear resistance of gray cast iron $[\mathrm{J}]$. Wear, 1991, 147(1): 195 206

12 Peter J. Blau. Mechanisms for transitional friction and wear
激 光

behavior of sliding metals $[\mathrm{J}]$. Wear, 1981, 72(1): 55 66

13 Wang Aiqin, Xie Jingpei, Wang Wenyan et al.. Effect of induction hardening on rolling wear properties of 45 steel[J]. Transactions of Materials and Heat Treatment, 2007, 28(1): $127 \sim 129$

王爱琴, 谢敬佩, 王文炎等. 表面感应淬火对 45 钢滚动磨损特 性的影响 [J]. 材料热处理学报, 2007, 28(1)：127 129

14 R. Colaco, R. Vilar. On the influence of retained austenite in the abrasive wear behaviour of a laser surface melted tool steel[J]. Wear, 2005, 258(1-4): 225 231

15 Zhang Qingmao, Liu Wenjin. Investigation on microstructures and tribological characteristic of laser remelting layers[J]. High Power Laser and Particle Beams, 2006, 18(3): 389 392

张庆茂, 刘文今. 激光熔凝层组织与摩擦学特性的研究 $[\mathrm{J}]$. 强 激光与粒子束, 2006, 18(3): 389 392

16 Sun Hao, Ling Gang, Li Hongwen et al. . Influence of scanning interval on microstructure and abrasive wear resistance of 45 steel by laser melting $[\mathrm{J}]$. Transactions of the Chinese Society of Agricultural Engineering, 2011, 27(2): 156 160

孙浩, 凌 刚, 李洪文等. 扫描间距对 45 钢激光熔凝强化组 织性能的影响 $[\mathrm{J}]$. 农业工程学报, 2011, 27(2): 156 160

17 K. L. Johnson. Contact Mechanics [M]. Xu Bingye, Luo Xuefu, Liu Beisheng. Transl. . Beijing: Higher Education Press, 1992. 56 59

K. L. Johnson. 接触力学 $[\mathrm{M}]$. 徐秉业, 罗学富, 刘倍声, 译. 北京: 高等教育出版社, 1992. 56 59

栏目编辑: 宋梅梅 